(Triticum aestivum): In vitro production and utilization of doubled haploids, p. 101-124. In: Y.P.S. Bajaj (ed.). Biotechnology in agriculture and forestry. vol. 12: Haploids in crop improvement I. Springer-Verlag, Berlin.

Powell, W., P.D.S. Caligari, and J.M. Dunwell. 1986. Field performance of lines derived from haploid and diploid tissues of Hordeum vulgare. Theor. Applied Genet. 72:458-465.

Reed, S.M. and E.A. Wernsman. 1989. DNA amplification among antherderived doubled haploid lines of tobacco and its relationship to agronomic performance. Crop Sci. 29:1072-1076.

401 Research Group, Laboratory of Plant Cell and Tissue Culture, Institute of Genetics, Academia Sinica. 1975. Primary study on induction of pollen plants of Zea mays. Acta Genet. Sin. 2:143.

Research Laboratory of Breeding. 1981. A preliminary study on the heredity and vitality of the progenies of tobacco pollen plants, p. 223-225. In: H. Hul (ed.). Plant tissue culture. Proc. Symp. Plant Tissue Culture, Peking, 1978. Pitman Publishing, London.

Rufty, R.C., E.A. Wernsman, C.E. Main, and G.V. Gooding, Jr. 1990. Registration of NC-BMR 42 and NC-BMR 90 germplasm lines of tobacco. Crop Sci. 30:241-242.

Schaeffer, G.W., F.T. Sharpe, Jr., and P.B. Cregan. 1984. Variation for improved protein and yield from rice anther culture. Theor. Applied Genet. 67:383-389.

Simmonds, N.W. 1979. Principles of crop improvement. Longman, London.

Thomas, E., F. Hoffman, and G. Wenzel. 1975. Haploid plantlets from microspores of rye. Z. Pflanzenzüchtg. 75:106-113.
Wang, Y., C. Sun, C. Wang, and N. Chien. 1973. The induction of the pollen plantlets of Triticale and Capsicum annuum from anther culture. Sci. Sinica 16:147-151.

Wenzel, G., F. Hoffmann, and E. Thomas. 1977. Anther culture as a breeding tool in rape. I. Ploidy level and phenotype of androgenetic plants. Z. Pflanzenzüchtg. 78:149-155.

Wenzel, G., O. Schieder, T. Przewozny, S.K. Sopory, and G. Melchers. 1979. Comparison of single cell culture derived Solanum tuberosum L. plants and a model for their application in breeding programs. Theor. Applied Genet. 55:49-55.

Winzeler, H., J. Schmid, and P.M. Fried. 1987. Field performance of androgenetic, doubled haploid spring wheat lines in comparison with lines selected by the pedigree system. Plant Breeding 99:41-48.

Wu, J. 1986. Breeding haploid corn by anther culture, p. 149-161. In: H. Hu and H. Yang (eds.). Haploids of higher plants in vitro. Springer-Verlag, Berlin.

Yang, X. and H. Fu. 1989. Hua-03 - A high protein indica rice. Intl. Rice Res. Nwsl. 14(3):14-15.

Zhu, D. and X. Pan. 1990. Rice (Oryza sativa L.): Guan 18-An improved variety through anther culture, p. 204-211. In: Y.P.S. Bajaj (ed.). Biotechnology in agriculture and forestry 2: Haploids in crop improvement I. Springer-Verlag, Berlin.

Zhao, Y., X. He, J. Wang, and W. Liu. 1990. Anther culture 28-A new diseaseresistant and high-yielding variety of winter wheat, p. 353-362. In: Y.P.S. Bajaj (ed.). Biotechnology in agriculture and forestry 13: Wheat. SpringerVerlag, Berlin.

\title{
Observations and Suggestions for Improving Somatic Hybridization by Plant Protoplast Isolation, Fusion, and Culture
}

\author{
Jude W. Grosser ${ }^{1}$ \\ Department of Horticultural Sciences, Citrus Research and Education Center, University of Florida, Institute of \\ Food and Agricultural Sciences, 700 Experiment Station Road, Lake Alfred, FL 33850
}

\begin{abstract}
Methods for the isolation and culture of Citrus protoplasts and subsequent plant regeneration are well developed (Grosser and Chandler, 1987; Grosser and Gmitter, 1990a; Kobayashi et al., 1983; Vardi and Galun, 1988; Vardi et al., 1982). Since the first report of somatic hybrid plant recovery in Citrus (Ohgawara et al., 1985), protoplast fusion techniques have been used to generate somatic hybrid plants from more than 30 parental combinations, including 25 from my laboratory (for reviews, see Gmitter et al., 1992; Grosser, 1991; Grosser and Gmitter 1990a, 1990b), and somatic cybrid plants from five combinations (Vardi et al., 1987, 1989). The comments and suggestions provided herein are based on my observations from research on Citrus, with lesser emphasis on Trifolium, but they may also be applicable to other plant protoplast systems, particularly woody species.
\end{abstract}

\section{Protoplast culture media development}

The complex 8P protoplast culture medium of Kao and Michayluk (1975) has been used for successful protoplast culture and plant regeneration of many plant species. The success of this complex medium is probably due to the appropriate concentrations of the multivitamin, organic acid, and sugar/alcohol additives that are combined with the basal medium formulation. These additives seem to provide additional buffering capacity and reduce the environmental stress on protoplasts by providing required metabolic intermediates.

Florida Agricultural Experiment Station Journal Series no. R-01899. The cost of publishing this paper was defrayed in part by the payment of page charges. Under postal regulations, this paper therefore must be hereby marked advertisement solely to indicate this fact.

${ }^{1}$ Associate Professor, plant cell genetics.
However, optimal basal tissue culture media have been developed for most plant genera, and an efficient protoplast culture medium may be developed for a particular genus by simply supplementing the optimal basal medium with $8 \mathrm{P}$ multivitamin, organic acid, and sugar/alcohol additives. This approach has been successful for Trifolium (Grosser and Collins, 1984; Myers et al., 1989) and Citrus (Grosser, 1991; Grosser and Gmitter, 1990a). Reducing or eliminating the ammonia content of the basal medium may also be useful. Most basal media contain high levels of $\mathrm{NH}_{4} \mathrm{NO}_{3}$ that can often be toxic to protoplasts. Glutamine or $\mathrm{Ca}\left(\mathrm{NO}_{3}\right)_{2}$ have been found to be good alternative sources of $\mathrm{N}$ in protoplast culture media, as demonstrated in BH3 Citrus protoplast medium (Grosser and Gmitter, 1990a) and Populus protoplast media (Russell and McCown, 1986).

Filter-sterilize all protoplast media, enzyme, and fusion solutions. Finally, use fresh protoplast culture media whenever possible, although protoplast culture media can be stored frozen or under refrigeration in brown bottles to prevent photo-oxidation.

\section{Protoplast isolation}

The importance of clean preparations of quality protoplasts cannot be overemphasized, and this starts with appropriate source material. Take leaves for protoplast isolation from vigorous and healthy plants grown under high-humidity and low-light conditions. I recommend using fully expanded, slightly hardened leaves from seedlings or from new flush of older plants. Growth chamber-grown leaves should be free of insecticide residue. When feathering leaves, use a sharp scalpel and avoid cutting near veins in larger, more developed leaves. Vacuum infiltration at 105 to $140 \mathrm{kPa}$ for 15 to $30 \mathrm{~min}$ is useful to increase enzyme penetration; leaves with compact parenchyma tissue may require more time in vacuum. Decontamination should not damage or 
dehydrate leaves (as indicated by burning or water soaking). Remove damaged sections of leaves before enzyme treatment. Grow genotypes with leaves that are over-sensitive to decontamination procedures in vitro to avoid this step.

Callus or suspension cultures used for protoplast isolation should be in the log phase of growth at the time of isolation. Friable tissue with low starch content generally gives the best results. Citrus embryogenic cultures can often require continual subculturing for long periods (often more than 1 year) before they reach adequate friability and appropriate starch levels for protoplast manipulation. Transferring Citrus callus to glutamine-containing media can sometimes reduce the starch content of cells to appropriate levels for protoplast isolation.

Use quality enzymes (e.g., cellulases, macerases, and pectolyases) whenever possible, as they liberate protoplasts more quickly and reduce exposure to harmful impurities. Desalting most pectinases, hemicellulases, and driselase before use may be beneficial. Combining an enzyme solution with a complex protoplast culture medium may reduce stress on protoplasts during isolation and thereby increase viability. Determine enzyme quality and quantity, time of exposure, and the concentration of osmoticum empirically. Overexposure to enzymes can weaken protoplast plasmalemmae and reduce viability. Excessive protoplast breakage can sometimes be reduced by increasing the concentration of the osmoticum. Gentle agitation by low-speed rotary shaking $(25-50 \mathrm{rpm})$ may facilitate protoplast liberation. Carry out isolations at a temperature that allows for high enzyme activity yet good protoplast viability (25-28C), and in darkness (most protoplasts are sensitive to light).

Purify protoplasts through a $45-\mu \mathrm{m}$ stainless steel or nylon filter to remove undigested cell clumps and large debris. Make reusable filters from the plastic reservoirs of Luer Lok 30-ml plastic syringes (Becton Dickinson \& Co., Rutherford, N.J.) and 45- $\mu \mathrm{m}$ stainless steel mesh (Gilson Co., Worthington, Ohio). Remove the upper $5 \mathrm{~cm}$ of the plastic reservoir with a hacksaw and melt it onto the stainless steel mesh using a hot plate. Trim excess mesh with sharp scissors. Resulting filters can withstand repeated autoclaving.

Particulate debris resulting primarily from broken protoplasts can reduce culture $\mathrm{pH}$, protoplast viability, and fusion frequency. Use a $25 \%$ sucrose : $13 \%$ mannitol or other gradient (Grosser and Gmitter, 1990a; Harms and Potrykus, 1978) to minimize this problem. I recommend this gradient for all protoplast preparations.

\section{Protoplast fusion}

The most successful methods used to generate somatic hybrid plants rely on the chemical polyethylene glycol (PEG) to induce protoplast adhesion and fusion. PEG is reliable, inexpensive, and nonphytotoxic to plant protoplasts if over-exposure is avoided. Historically, high-molecular-weight PEG (MW 6000-8000) has been used, but more recently, Chand et al. (1988) reported that $1500 \mathrm{MW}$ (Aldrich Chemical Co., Milwaukee) was more efficient. I recommend the procedure of Menczel et al. (1981), as modified by Grosser and Gmitter (1990a). Monitor the $\mathrm{pH}$ of prepared PEG solutions weekly, because they acidify rapidly. Because smaller protoplasts fuse at lower frequencies than larger ones, use equal volumes rather than equal densities of protoplasts from each parental source to maximize fusion frequency. Protoplast density also effects fusion frequency and culturability; higher densities can reduce fusion frequency. Adjust protoplast densities on a pellet: medium (v/v) basis following centrifugation of mixed protoplasts (Grosser and Gmitter, 1990a). The fusion of callus- or suspension-derived protoplasts with leaf-derived protoplasts facilitates fusion frequency determinations. Fusions of this combination also seem to result in enhanced somatic embryogenesis and subsequent plant recovery. Add PEG solution immediately after plating out mixed protoplasts, before parental types segregate according to buoyancy. Adjust the duration of exposure to PEG and elution solutions to achieve a balance between fusion frequency and postfusion protoplast viability. Genotypes that liberate fragile protoplasts require shorter exposure times. Repeat experiments over several consecutive days, with continual observation and adjustment. Somatic hybridization is a "numbers game"; therefore, generate a large number of fusion cultures for each attempted parental combination.

\section{Protoplast culture}

Culture protoplasts in a manner that maximizes gas exchange (thinlayered, semisolid media or shallow liquid media). Seal fusion cultures tightly with Nescofilm (Karlan Chemical Corp., Torrance, Calif.) or other appropriate film to avoid desiccation; media containing mannitol are particularly susceptible to desiccation. Maintain high humidity during culture and osmoticum reduction.

For Citrus, I have observed that extremely high plating efficiencies can inhibit the induction of somatic embryogenesis and subsequent plant regeneration. If high plating efficiencies are observed early during the culture cycle (first 4-8 weeks), dilute the culture or transfer cultures to solid medium early (Grosser and Gmitter, 1990a). Transfer callus or somatic embryos recovered following protoplast culture regularly and do not leave them on the same medium-plates for more than 4 weeks. Somatic embryos (including those with distorted shapes) that do not yield plants following several passages on germination media can sometimes be induced to regenerate plants via adventive shoot-bud induction upon transfer to cytokinin-containing media (Grosser and Gmitter, 1990a). In most cases, plants can eventually be recovered from semi-organized green tissue.

Establish efficient plant regeneration from protoplasts for at least one parent in each desired combination before attempting somatic hybridization. In my opinion, sophisticated somatic hybrid selection schemes have been over-emphasized, often at the expense of plant regeneration. The use of metabolic inhibitors, irradiation, deficient mutants, protoplast labeling, and flow cytometry, or micromanipulation may often be avoided by achieving efficient protoplast fusion and plant regeneration. In Citrus, somatic hybrid combinations are generally competitive, and somatic hybrid plants can be recovered even when populations of regenerated plants consist primarily of parental types from unfused protoplasts (Tusa et al., 1990).

\section{Literature Cited}

Chand, P.K., M.R. Davey, J.B. Power, and E.C. Cocking. 1988. An improved procedure for protoplast fusion using polyethylene glycol. J. Plant Physiol. 133:480-485.

Gmitter, F.G., Jr., J.W. Grosser, and G.A. Moore. 1992. Citrus, p. 335-370. In: R.E. Litz and F. Hammerschlag (eds.). Biotechnology of perennial fruit crops. CAB International, Oxon, U.K.

Grosser, J.W. 1994. In vitro culture of tropical fruits, p. 475-496. In: I.K. Vasil and T.A. Thorpe (eds.). Plant cell and tissue culture. Kluwer Academic Publishers, Dordrecht, The Netherlands.

Grosser, J.W. and J.L. Chandler. 1987. Aseptic isolation of leaf protoplasts from Citrus, Poncirus, Citrus $\times$ Poncirus hybrids and Severinia for use in somatic hybridization experiments. Scientia Hort. 31:253-257.

Grosser, J.W. and G.B. Collins. 1984. Isolation and culture of Trifolium rubens protoplasts with whole plant regeneration. Plant Sci. Lett. 37:165-170.

Grosser, J.W. and F.G. Gmitter, Jr. 1990a. Protoplast fusion and citrus improvement. Plant Breeding Rev. 8:339-374.

Grosser, J.W. and F.G. Gmitter, Jr. 1990b. Wide hybridization of Citrus via protoplast fusion: Progress, strategies and limitations, p. 31-41. In: A.B. Bennett and S.D. O'Neill (eds.). Horticultural biotechnology. Plant biology II. Wiley-Liss, New York.

Harms, C.T. and I. Potrykus. 1978. Fractionation of plant protoplasts types by iso-osmotic density gradient centrifugation. Theor. Applied Genet. 53:5763.

Kao, K.N. and M.R. Michayluk. 1975. Nutritional requirements for growth of Vicia hajastana cells and protoplasts at a very low population density in liquid media. Planta 126:105-110.

Kobayashi, S., H. Uchimaya, and I. Ikeda. 1983. Plant regeneration from 'Trovita' orange protoplasts. Jpn. J. Breeding 33:119-122.

Menczel, L., F. Nagy, Z. Kiss, and P. Maliga. 1981. Streptomycin resistant and sensitive hybrids of Nicotiana tabacum and Nicotiana knightiana: Correlation of resistance with N. tabacum plastids. Theor. Applied Genet. 53:5763.

Myers, J.R., J.W. Grosser, N.L. Taylor, and G.B. Collins. 1989. Genotype dependent whole plant regeneration from protoplasts of red clover (Trifolium pratense L.). Plant, Cell, Tissue Organ Culture 19:113-127.

Ohgawara, T., S. Kobayashi, E. Ohgawara, H. Uchimaya, and S. Ishu. 1985. Somatic hybrid plants obtained by protoplast fusion between Citrus sinensis and Poncirus trifoliata. Theor. Applied Genet. 71:1-4.

Russell, J.A. and B.H. McCown. 1986. Culture and regeneration of Populus leaf protoplasts isolated from non-seedling tissue. Plant Sci. 46:133-142. 
Tusa, N., J.W. Grosser, and F.G. Gmitter, Jr. 1990. Plant regeneration of 'Valencia' sweet orange, 'Femminello' lemon, and the interspecific somatic hybrid following protoplast fusion. J. Amer. Soc. Hort. Sci. 115:10431046.

Vardi, A., P. Arzee-Gonen, A. Frydman-Shani, and S. Bleichman. 1989. Protoplast fusion mediated transfer of organelles from Microcitrus and Citrus and regeneration of novel alloplasmic trees. Theor. Applied Genet. 78:741-747.
Vardi, A., A. Breiman, and E. Galun. 1987. Citrus cybrids: Production by donor-recipient protoplast-fusion and verification by mitochondrial-DNA restriction profiles. Theor. Applied Genet. 75:51-58.

Vardi, A. and E. Galun. 1988. Recent advances in protoplast culture of horticultural crops: Citrus. Scientia Hort. 37:217-230.

Vardi, A., P. Spiegel-Roy, and E. Galun. 1982. Plant regeneration from Citrus protoplasts: Variability in methodological requirements among cultivars and species. Theor. Applied Genet. 62:171-176.

\title{
A Review of Plant Embryo Culture
}

\author{
Mark P. Bridgen \\ Department of Plant Science, U-67, 1376 Storrs Road, University of Connecticut, Storrs, CT 06269
}

Tapping germplasm resources to improve cultivated plants depends on introducing natural variability through traditional and biotechnological breeding methods. Intervarietal and interspecific crosses, followed by selection, have accounted for the improvement in quality and yield potential of practically all major crops (Raghavan, 1986). One biotechnological technique that has been beneficial is embryo culture.

Embryo culture involves isolating and growing an immature or mature zygotic embryo under sterile conditions on an aseptic nutrient medium with the goal of obtaining a viable plant. The basic premise for this technique is that the integrity of the hybrid genome is retained in a developmentally arrested or an abortive embryo and that its potential to resume normal growth may be realized if supplied with the proper growth substances. The technique depends on isolating the embryo without injury, formulating a suitable nutrient medium, and inducing continued embryogenic growth and seedling formation.

The culture of immature embryos is used to rescue embryos that would normally abort or that would not undergo the progressive sequence of ontogeny. This process is difficult due to the tedious dissection necessary and the complex nutrient medium requirements. Success with this type of culture depends strongly on the developmental stage of the embryo when it is isolated (Monnier, 1978; Raghavan, 1980).

The culture of mature embryos from ripened seeds is used to eliminate seed germination inhibitors or to shorten the breeding cycle if, for example, dormancy is a problem. This culture is easy and only requires a simple nutrient medium with agar, sugar, and minerals.

\section{HISTORY}

Embryo culture, sometimes called embryo rescue, is an in vitro technique that has been used for more than half a century to save the hybrid products of fertilization when they might otherwise degenerate. Success was first achieved in 1904 by Hannig who obtained viable plants from mature embryos of two crucifers that were isolated aseptically and grown on a mineral salt medium supplemented with sugar (Norstog, 1979). In 1924, Dietrich cultured mature and immature embryos of various plant species to determine whether they could still germinate without completing the dormancy period. He reported that the mature embryos grew immediately, circumventing dormancy. The immature embryos germinated precociously without further embryo development. Laibach first described zygotic embryo culture for interspecific hybridization in 1925 . He observed that seeds from interspecific crosses between Linum perenne L. x Linum austriacum L. were nonviable; however, if embryos were excised early during seed development and cultured in vitro, then embryo abortion was overcome. Later, van Overbeek et al. (1941) discovered that small Datura hybrid embryos could be grown in culture on media containing

Storrs Agricultural Experiment Station no. 1503. The cost of publishing this paper was defrayed in part by the payment of page charges. Under postal regulations, this paper therefore must be hereby marked advertisement solely to indicate this fact. coconut milk. This discovery ultimately led to understanding the importance of reduced $\mathrm{N}$ in the form of amino acids for embryo culture.

Since the early 1940s, embryo culture has been used increasingly to understand the physical and nutritional requirements for embryonic development, bypass seed dormancy, shorten the breeding cycle, test seed viability, provide material for micropropagation, and rescue immature hybrid embryos from incompatible crosses (Hu and Wang, 1986).

\section{APPLICATIONS}

Embryo culture is one of the earliest forms of in vitro culture applied to practical problems and is probably the tissue culture technique that has proven of greatest value to breeders (Dunwell, 1986). Its major application in plant breeding has been for interspecific hybridization.

Many unsuccessful crosses result from embryo abortion. Early embryo abortion occurs primarily because the endosperm fails to develop properly (Hu and Wang, 1986). With interspecific crosses, intergeneric crosses, and crosses between diploids and tetraploids, the endosperm often develops poorly or not at all. By aseptically culturing the embryo in a nutrient medium, this problem may be overcome. Embryos of some nonviable hybrids may possess the potential for initiating development by avoiding postzygotic barriers within the mother plant. Several successful cases have been documented with embryos arising from interspecific hybrids and intergeneric hybrids (Ramming, 1990; Sharma and Gill, 1983; Williams, 1980; Williams and De Lautour, 1980; Williams et al., 1982).

Embryo culture can shorten the breeding cycle by overcoming dormancy in seeds. Dormancy may be caused by endogenous inhibitors, light requirements, low temperatures, dry storage requirements, and embryo immaturity (Yeung et al., 1981). Seed dormancy factors may be localized in the seedcoat, the endosperm, or both. By removing the embryos from the influences of these factors, the embryos germinate and grow quickly and the breeding cycle is shortened. Isolated embryos can also be vernalized and may, in some instances, reduce the generation time by 40 days (Sharma and Gill, 1983).

In addition to the applied uses of embryo culture, the procedure is useful in basic studies. Growing embryos outside the ovule (ex ovulo) is an excellent way to study the nutrition and metabolism of the embryos at various stages of development. The technique can also be used to examine the growth requirements of embryos, the effects of phytohormones and environmental conditions on zygotic embryogenesis, and the regeneration potentials of whole embryos and their segments (Yeung et al., 1981). Embryo culture can be used to localize sites of germination promoters and inhibitors, for studies of embryogenesis, and for cryopreservation (Grout, 1986).

Embryo culture can be used to produce haploids through eliminating chromosomes following distant hybridization. This can occur by rescuing haploid maternal embryos in which the paternal chromosomes have been eliminated. In these situations, fertilization occurs, but the pollen parent chromosomes are subsequently eliminated by the 